\title{
ON A CLASS OF UNIVALENT, STAR SHAPED MAPPINGS
}

\section{ALBERT SCHILD ${ }^{1}$}

1. Introduction. Among all functions $w=f(z)=z+\sum_{n=2}^{\infty} a_{n} z^{n}$ regular and univalent in the unit circle, two classes of functions have been discussed extensively: The class of functions mapping the unit circle onto star shaped regions, characterized by $\operatorname{Re}\left\{z f^{\prime}(z) / f(z)\right\} \geqq 0$ for $|z|<1$, and the class of functions mapping the unit circle onto convex regions, characterized by $\operatorname{Re}\left\{z f^{\prime \prime}(z) / f^{\prime}(z)\right\}+1 \geqq 0$ for $|z|<1$.

This short paper will examine some of the geometric and analytic properties of a class of functions $w=f(z)=z+\sum_{n=2}^{\infty} a_{n} z^{n}$ which map the unit circle onto a region whose geometric nature is somewhat intermediate between star shaped and convex. The functions under consideration are to satisfy $\operatorname{Re}\left\{z f^{\prime}(z) / f(z)\right\} \geqq 1 / 2$ for all $|z|<1$.

Interest in functions of this type can be traced back to two papers by A. Marx [4] and E. Strohäcker [8] who have shown that for any function $w=f(z)=z+\sum_{n=2}^{\infty} a_{n} z^{n}$, which maps the unit circle onto a convex region, we have $\operatorname{Re}\left\{z f^{\prime}(z) / f(z)\right\} \geqq 1 / 2$, and as the function $f(z)=z /(1+z)$ shows, the constant $1 / 2$ cannot be improved. It is also clear that the converse is not true, i.e. functions for which $\operatorname{Re}\left\{z f^{\prime}(z) / f(z)\right\} \geqq 1 / 2$ need not map the unit circle onto a convex region. An example of this type is given by the function $w=f(z)$ $=z-1 / 3 z^{2}$ for which $\operatorname{Re}\left\{z f^{\prime}(z) / f(z)\right\} \geqq 1 / 2,|z| \leqq 1$, yet the image region is not convex.

Recently, interest in this class of functions was roused again in a paper by R. F. Gabriel [2]. There it is shown that if $p(z)$ is analytic and single valued for $|z|<1$, and if we denote by $w_{1}(z)=1+\sum_{n=2}^{\infty} a_{n} z^{n}$ and by $w_{2}(z)=z+\sum_{n=2}^{\infty} b_{n} z^{n}$ two linearly independent solutions of $w^{\prime \prime}+p(z) w=0$, then $f(z)=w_{1}(z) / w_{2}(z)=1 / z+\cdots$ will map $|z| \leqq 1$ onto the exterior of a convex region if and only if $\operatorname{Re}\left\{z f^{\prime}(z) / f(z)\right\} \geqq 1 / 2$ for $|z|<1$.

M. S. Robertson [6] considered also the class of functions $w=f(z)$ $=z+\sum_{n=2}^{\infty} a_{n} z^{n}$ for which $\operatorname{Re}\left\{z f^{\prime}(z) / f(z)\right\} \geqq \alpha>0,|z|<1$.

Notation. On the following pages we shall denote the class of functions $w=f(z)=z+\sum_{n=2}^{\infty} a_{n} z^{n}$ regular and univalent in the unit circle by $S$.

The subclass of $S$ which map $|z|<1$ onto a star shaped region, i.e. for which $\operatorname{Re}\left\{z f^{\prime}(z) / f(z)\right\} \geqq 0$ by St.

Presented to the Society, January 28, 1958; received by the editors March 7, 1958 and, in revised form, April 21, 1958.

1 This work was aided by a grant from the Committee on Research and Publications of Temple University. 
The subclass of St which map $|z|<1$ onto a "special" star shaped region, i.e. for which $\operatorname{Re}\left\{z f^{\prime}(z) / f(z)\right\} \geqq 1 / 2$ by St*.

The subclass of $S$ which map $|z|<1$ onto a convex region, i.e. for which $\operatorname{Re}\left\{z f^{\prime \prime}(z) / f^{\prime}(z)\right\}+1 \geqq 0$ by $C$.

By [4] and [8] we obviously have:

$$
S \supset \mathrm{St} \supset \mathrm{St}^{*} \supset C \text {. }
$$

2. Methods for constructing functions of class $\mathrm{St}^{*}$. The intermediacy of St* between St and C suggests, that one might obtain functions of class $\mathrm{St}^{*}$ by "relaxing" the conditions on functions of class $\mathrm{C}$ or by "strengthening" conditions on functions of class St. Both methods yield results.

Theorem 2.1. If $w=f(z) \in C$, then $g(z)=z\left(f^{\prime}(z)\right)^{\alpha} \in \mathrm{St}^{*}$ for $0<\alpha$ $\leqq 1 / 2$. The converse is true for $\alpha=1 / 2$.

Proof. $\operatorname{Re}\left\{z g^{\prime}(z) / g(z)\right\}=\operatorname{Re}\left\{1+\alpha z f^{\prime \prime}(z) / f^{\prime}(z)\right\} \geqq 1-\alpha \geqq 1 / 2$. For the converse we have: $\operatorname{Re}\left\{z f^{\prime \prime}(z) / f^{\prime}(z)\right\}+1=\operatorname{Re}\left\{2 z g^{\prime}(z) / g(z)\right\}-1 \geqq 0$. This should be compared with the well known result that if $f(z) \in C$ then $g(z)=z f^{\prime}(z) \in \mathrm{St}$ and conversely.

Corollary 2.2. Let $\left|b_{i}\right|=1$ and $\sum_{i=1}^{n} \mu_{i} \leqq 1, \mu_{i} \geqq 0$, then $w=g(z)$ $=z \prod_{i=1}^{n}\left(1-b_{i} z\right)^{-\mu_{i}} \in \mathrm{St}^{*}$.

Proof. $f(z)=\int_{0}^{z} \prod_{i=1}^{n}\left(1-b_{i} t\right)^{-v_{i}} d t \in C$ if $v_{i} \geqq 0$ and $\sum_{i=1}^{n} v_{i} \leqq 2$, $\left|b_{i}\right|=1$. The corollary follows if we let $\alpha=1 / 2$ in Theorem 2.1.

Theorem 2.3. If $w=f(z) \in \mathrm{St}$, then $g(z)=z\{f(z) / z\}^{\alpha} \in \mathrm{St}^{*}$ for $0<\alpha \leqq 1 / 2$. The converse is true for $\alpha=1 / 2$.

Proof. $\operatorname{Re}\left\{z g^{\prime}(z) / g(z)\right\}=\operatorname{Re}\left\{\alpha z f^{\prime}(z) / f(z)+1-\alpha\right\} \geqq 1-\alpha \geqq 1 / 2$.

For the converse we have: $\operatorname{Re}\left\{z f^{\prime}(z) / f(z)\right\}=\operatorname{Re}\left\{2 z g^{\prime}(z) / g(z)-1\right\}$ $\geqq 0$. (Marx [4] has the above theorem for $\alpha=1 / 2$.)

The method of Theorem 2.3 for constructing functions of class $\mathrm{St}^{*}$ gives some insight into the geometrical nature of the region onto which the unit circle is mapped by functions of class St*. If $z=r e^{i \theta}$ is mapped by $w=f(z) \in \mathrm{St}$ into $\mathrm{Re}^{i \phi}$, then a comparison of $r$ and $R$ and $\theta$ and $\phi$ tells us about the amount of distortion. We notice that $g(z)=\{z f(z)\}^{1 / 2} \in \mathrm{St}{ }^{*}$ reduces the amount of distortion effected by $f(z) \in$ St by an "averaging" process, i.e. $z=r e^{i \theta}$ will be mapped by $g(z)$ in to $\rho e^{i \psi}$ where $\rho=(r R)^{1 / 2}$ and $\psi=(\theta+\phi) / 2$.

\section{Distortion theorems for functions of class St*.}

Theorem 3.1. For all $g(z) \in \mathrm{St}^{*}$ we have $|z| /(1+|z|) \leqq|g(z)|$ $\leqq|z| /(1-|z|)$. 
Proof. By Theorem $2.3(\alpha=1 / 2)$ and the "Verzerrungs Satz" we have: $|z| /(1+|z|)^{2} \leqq\left|g^{2}(z) / z\right| \leqq|z| /(1-|z|)^{2}$ and hence the Theorem follows. These inequalities are sharp for $g(z)=z /(1+z) \in \mathrm{St}^{*}$, $z= \pm r$.

Theorem 3.2. For all $g(z) \in \mathrm{St}^{*}$ the domain of values of $z g^{\prime}(z) / g(z)$ is the circle with center at $1 /\left(1-|z|^{2}\right)$ and radius $|z| /\left(1-|z|^{2}\right)$.

Proof. Let

$$
G(z)=z g^{\prime}(z) / g(z)-1 / 2
$$

and

$$
H(z)=(2 G(z)-1) /(2 G(z)+1)=\left\{z g^{\prime}(z) / g(z)-1\right\} / z g^{\prime}(z) / g(z) .
$$

Then $H(z)$ is regular for $|z|<1, H(0)=0$ and $|H(z)|<1$ for $|z|<1$. Hence the Lemma of Schwarz can be applied and we have for $|z|<1$

$$
\left|\left(\frac{z g^{\prime}(z)}{g(z)}-1\right) / \frac{z g^{\prime}(z)}{g(z)}\right|<|z| \text { or: }\left|\frac{z g^{\prime}(z)}{g(z)}-1\right|<|z|\left|\frac{z g^{\prime}(z)}{g(z)}\right| \text {. }
$$

But the domain defined by this inequality is the interior of the Circle of Apollonius with the line segment form $1 /(1+|z|)$ to $1 /(1-|z|)$ as a diameter, i.e. the interior of the circle with radius $|z| /\left(1-|z|^{2}\right)$ and center at $1 /\left(1-|z|^{2}\right)$. The function $f(z)=z /(1+z) \in \mathrm{St}^{*}$ shows that the theorem cannot be improved.

4. Some coefficient relations. It is well known that if $f(z) \in S t$ then $\left|a_{n}\right| \leqq n$. For functions of class $\mathrm{St}^{*}$ we have:

THEOREM 4.1. If $f(z)=z+\sum_{n=2}^{\infty} a_{n} z^{n} \in \mathrm{St}^{*}$, then $\left|a_{n}\right| \leqq 1$.

Proof. Let $p(z)=2 z f^{\prime}(z) / f(z)-1=1+c_{1} z+c_{2} z^{2}+\cdots$. Since $p(z)$ is regular and $\operatorname{Re}\{p(z)\}>0$ for $|z|<1$, therefore, by a well known lemma we have $\left|c_{n}\right| \leqq 2$ for $n=1,2,3, \cdots$.

Comparing coefficients we obtain $2(n-1) a_{n}=c_{n-1}+a_{2} c_{n-2}+\cdots$ $+a_{n-1} c_{1}$, and hence: $\left|a_{n}\right| \leqq 1 /(n-1)\left\{1+\left|a_{2}\right|+\cdots+\left|a_{n-1}\right|\right\}$. It follows now by induction that $\left|a_{n}\right| \leqq 1$ for $n=1,2,3, \cdots$.

The function $f(z)=z /(1-z)=\sum_{n=1}^{\infty} z^{n} \in \mathrm{St}^{*}$ shows that these inequalities are sharp.

For some kind of a converse we have:

Theorem 4.2. If $g(z)=z+\sum_{n=2}^{\infty} a_{n} z^{n}$ and if $\sum_{n=2}^{\infty}(2 n-1)\left|a_{n}\right| \leqq 1$ then $g(z) \in \mathrm{St}^{*}$.

Proof. The proof is based on a method used by A. Goodman [3]. We have: 


$$
\begin{aligned}
\frac{z g^{\prime}(z)}{g(z)}-\frac{1}{2} & =\frac{1+3 a_{2} z+5 a_{3} z^{2}+\cdots+(2 n-1) a_{n} z^{n-1}+\cdots}{2\left(1+a_{2} z+a_{3} z^{2}+\cdots+a_{n} z^{n-1}+\cdots\right.} \\
& =\frac{1}{2}+\sum_{n=1}^{\infty} b_{n} z^{n}
\end{aligned}
$$

where

$$
\begin{aligned}
& b_{1}=a_{2} . \\
& b_{2}=2 a_{3}-a_{2} b_{1}, \\
& b_{3}=3 a_{4}-a_{2} b_{2}-a_{3} b_{1}, \\
& \vdots \\
& \dot{b}_{n-1}=(n-1) a_{n}-a_{2} b_{n-2}-a_{3} b_{n-3}-\cdots-a_{n-1} b_{1},
\end{aligned}
$$

and therefore for $n \geqq 2$

$$
\sum_{k=1}^{n-1} b_{k}=\sum_{k=2}^{n}(k-1) a_{k}-a_{2} \sum_{k=1}^{n-2} b_{k}-a_{3} \sum_{k=1}^{n-3} b_{k}-\cdots-a_{n-1} b_{1} .
$$

The inequality of the theorem implies that $\left|b_{1}\right|=\left|a_{2}\right| \leqq 1 / 3$. It will now be shown by mathematical induction that for all $n$ we have $\left|\sum_{k=1}^{n} b_{k}\right| \leqq 1 / 2$. Assume that $\left|\sum_{k=1}^{m} b_{k}\right| \leqq 1 / 2$ for $m=1,2,3, \cdots$, $n-2$. Then (4.5) yields

$$
\begin{aligned}
\left|\sum_{k=1}^{n-1} b_{k}\right| & \leqq \sum_{k=2}^{n}(k-1)\left|a_{k}\right|+\frac{1}{2} \sum_{k=2}^{n-1}\left|a_{k}\right| \leqq \sum_{k=2}^{n}(k-1 / 2)\left|a_{k}\right| \\
& =\frac{1}{2} \sum_{k=2}^{n}(2 k-1)\left|a_{k}\right| \leqq 1 / 2
\end{aligned}
$$

by the inequality of the theorem, and hence $\left|\sum_{k=1}^{n} b_{k}\right| \leqq 1 / 2$ for all $n$. From (4.3) it follows now that

$$
\left|z g^{\prime}(z) / g(z)-1\right| \leqq 1 / 2 \text { for } z=r .
$$

But the inequality of Theorem 4.2 and the special starshapedness of the image domain are invariant under rotations of the $z$ and $w$ planes. Hence any point in the unit circle may be placed in the interval $[0,1]$, and thus $(4.7)$ is valid throughout the unit circle i.e.

$$
\operatorname{Re}\left\{z g^{\prime}(z) / g(z)\right\} \geqq 1 / 2 \text { for }|z|<1 .
$$

COROLlARY 4.8. Let $g(z)=z-\sum_{n=2}^{\infty} a_{n} z^{n}$, where all $a_{i} \geqq 0$, then $g(z)$ $\in \mathrm{St}^{*}$, if and only if $\sum_{n=2}^{\infty}(2 n-1) a_{n} \leqq 1$. 
Proof. The sufficiency of the condition follows from the Theorem. For the necessity we have:

$$
\operatorname{Re}\left\{\frac{z g^{\prime}(z)}{g(z)}-\frac{1}{2}\right\}=\operatorname{Re}\left\{\frac{1-\sum_{n=2}^{\infty}(2 n-1) a_{n} z^{n-1}}{2\left(1-\sum_{n=2}^{\infty} a_{n} z^{n-1}\right)}\right\} .
$$

If $\sum_{n=2}^{\infty}(2 n-1) a_{n}>1$, then we could find a positive value of $z, r_{0}$, for which the numerator of (4.9) would be negative and the denominator positive and hence $\operatorname{Re}\left\{z g^{\prime}(z) / g(z)\right\}<1 / 2$ for that value of $z$ and all positive values of $z>r_{0}$. (See also [7].)

5. The radius of special star shapedness. We define the radius of special star shapedness, $r^{*}$, as the upper bound of the radii, $r$, of circles $|z| \leqq r$, which are mapped by any function $f(z) \in S$ onto a region of special star shapedness, i.e. that for all functions $f(z) \in S$ we have $\operatorname{Re}\left\{z f^{\prime}(z) / f(z)\right\} \geqq 1 / 2$ for all $|z| \leqq r$. From the introduction it follows that $r^{*}$ is larger than the bound of convexity (Rundungsschranke) and less than the bound of starlikeness. Therefore

$$
2-3^{1 / 2}=.268 \cdots \leqq r^{*} \leqq .65 \cdots=\tanh \pi / 4 .
$$

We have:

Theorem 5.2. $r^{*}=1 / 3$ for all $f(z) \in \mathrm{St}$, and hence $r^{*} \leqq 1 / 3$.

Proof. By [5] we have for any $f(z) \in \mathrm{St}$

$$
\frac{1-|z|}{1+|z|} \leqq \operatorname{Re}\left\{\frac{z f^{\prime}(z)}{f(z)}\right\} \leqq \frac{1+|z|}{1-|z|} .
$$

Therefore $\operatorname{Re}\left\{z f^{\prime}(z) / f(z)\right\} \geqq(1-|z|) /(1+|z|) \geqq 1 / 2$ for all $|z| \leqq 1 / 3$. This result is sharp for $f(z)=z(1+z)^{-2} \in \mathrm{St}$ and $z=1 / 3$.

TheOREM 5.3. A lower bound for $r^{*}$ is: $r^{*}>.301 \cdots$.

Proof. If we let

$$
\begin{aligned}
g(z) & =f^{-1 / 2}\left(z^{-2}\right) \\
& =z+b_{1} / z+b_{3} / z^{3}+\cdots+b_{2 n-1} / z^{2 n-1}+\cdots
\end{aligned}
$$

then by Bieberbach's Flächensatz [1] we have

$$
\sum_{n=1}^{\infty}(2 n-1)\left|b_{2 n-1}\right|^{2} \leqq 1 .
$$

The condition $\operatorname{Re}\left\{z f^{\prime}(z) / f(z)\right\} \geqq 1 / 2$ is equivalent to 


$$
\left|\frac{z f^{\prime}(z)}{f(z)}\right| \geqq\left|\frac{z f^{\prime}(z)}{f(z)}-1\right|
$$

i.e. by (5.4)

$$
\left|\frac{z^{-1 / 2} g^{\prime}\left(z^{-1 / 2}\right)}{g\left(z^{-1 / 2}\right)}\right| \geqq\left|\frac{z^{-1 / 2} g^{\prime}\left(z^{-1 / 2}\right)}{g\left(z^{-1 / 2}\right)}-1\right|
$$

or :

$$
\begin{aligned}
& \left|\frac{1-b_{1} z-3 b_{3} z^{2}-\cdots-(2 n-1) b_{2 n-1} z^{n}-\cdots}{1+b_{1} z+b_{3} z^{2}+\cdots+b_{2 n-1} z^{n}+\cdots}\right| \\
& \geqq\left|\frac{1-b_{1} z-3 b_{3} z^{2}-\cdots-(2 n-1) b_{2 n-1} z^{n}+\cdots}{1+b_{1} z+b_{3} z^{2}+\cdots+b_{2 n-1} z^{n}+\cdots}-1\right|
\end{aligned}
$$

or:

$$
\begin{aligned}
& \left|1-b_{1} z-3 b_{3} z^{2}-\cdots-(2 n-1) b_{2 n-1} z^{n}\right| \\
& \quad \geqq 2\left|b_{1} z+2 b_{3} z^{2}+3 b_{5} z^{3}+\cdots+n b_{2 n-1} z^{n}+\cdots\right| .
\end{aligned}
$$

A sufficient condition for this inequality to be satisfied is that

$$
\begin{aligned}
1-\left|b_{1}\right| r-3\left|b_{3}\right| r^{2}-\cdots-(2 n-1) & \left|b_{2 n-1}\right| r^{n}+\cdots \\
& \geqq 2\left|b_{1}\right| r+4\left|b_{3}\right| r^{2}+\cdots
\end{aligned}
$$

or:

$3\left|b_{1}\right| r+7\left|b_{3}\right| r^{2}+11\left|b_{5}\right| r^{3}+\cdots$

$$
+(4 n-1)\left|b_{2 n-1}\right| r^{n}+\cdots \leqq 1
$$

or

$$
\begin{aligned}
2\left\{\left|b_{1}\right| r+3\left|b_{3}\right| r^{2}+\cdots+(2 n-1)\left|b_{2 n-1}\right| r^{n}+\cdots\right\} \\
+\left\{\left|b_{1}\right| r+\left|b_{3}\right| r^{2}+\cdots+\left|b_{2 n-1}\right| r^{n}+\cdots\right\} \leqq 1
\end{aligned}
$$

If in the first parenthesis of inequality (5.8) welet $c_{n}=(2 n-1)^{1 / 2}\left|b_{2 n-1}\right|$ and $d_{n}=(2 n-1)^{1 / 2} \cdot r^{n}$ then the Inequality of Schwarz

$$
\sum c_{n} \cdot d_{n} \leqq\left(\sum c_{n}^{2}\right)^{1 / 2} \cdot\left(\sum d_{n}^{2}\right)^{1 / 2}
$$

gives

$$
\begin{aligned}
& \left|b_{1}\right| r+3\left|b_{3}\right| r^{2}+\cdots+(2 n-1)\left|b_{2 n-1}\right| r^{n}+\cdots \\
& \quad \leqq\left(\left|b_{1}\right|^{2}+3\left|b_{3}\right|^{2}+\cdots+(2 n-1)\left|b_{2 n-1}\right|^{2}+\cdots\right)^{1 / 2} \\
& \quad \cdot\left(r^{2}+\cdots+(2 n-1) r^{2 n}+\cdots\right)^{1 / 2}
\end{aligned}
$$

and therefore by (5.5) 


$$
\begin{aligned}
& \left|b_{1}\right| r+3\left|b_{3}\right| r^{2}+\cdots+(2 n-1)\left|b_{2 n-1}\right| r^{n}+\cdots \\
& \quad \leqq\left(r^{2}+3 r^{4}+\cdots+(2 n-1) r^{2 n}+\cdots\right)^{1 / 2}=\frac{r\left(1+r^{2}\right)^{1 / 2}}{1-r^{2}}
\end{aligned}
$$

Similarly, if in the second parenthesis of inequality (5.8) we let $c_{n}=(2 n-1)^{1 / 2}\left|b_{2 n-1}\right|, d_{n}=r^{n}(2 n-1)^{-1 / 2}$ and apply the Inequality of Schwarz again, we get:

$$
\begin{gathered}
\left|b_{1}\right| r+\left|b_{3}\right| r^{2}+\cdots+\left|b_{2 n-1}\right| r^{n}+\cdots \\
\leqq \\
\left(\left|b_{1}\right|^{2}+3\left|b_{3}\right|^{2}+\cdots+(2 n-1)\left|b_{2 n-1}\right|+\cdots\right)^{1 / 2} \\
\quad\left(r^{2}+\frac{r^{4}}{3}+\cdots+\frac{r^{2 n}}{2 n-1}+\cdots\right)^{1 / 2}
\end{gathered}
$$

and again by (5.5)

$$
\begin{aligned}
& \left|b_{1}\right| r+\left|b_{3}\right| r^{2}+\cdots+\left|b_{2 n-1}\right| r^{n}+\cdots \\
& \leqq\left(r^{2}+\frac{r^{4}}{3}+\cdots+\frac{r^{2 n}}{2 n-1}+\cdots\right)^{1 / 2}=\left(\frac{r}{2} \ln \frac{1+r}{1-r}\right)^{1 / 2}
\end{aligned}
$$

Substituting (5.10) and (5.12) into (5.8) we have:

$$
\frac{2 r\left(1+r^{2}\right)^{1 / 2}}{1-r^{2}}+\left(\frac{r}{2} \log \frac{1+r}{1-r}\right)^{1 / 2}<1 .
$$

This will be satisfied for $|z|=r<.301 \cdots$, i.e. $r^{*}>.301 \cdots$.

\section{BIBLIOGRAPHY}

1. L. Bieberbach, Lehrbuch der Funktionentheorie, vol. 2, pp. 84-85.

2. R. F. Gabriel, The Schwarzian derivative and convex functions, Proc. Amer. Math. Soc. vol. 6 (1955) pp. 58-66.

3. A. Goodman, Univalent functions and nonanalytic curves, Proc. Amer. Math. Soc. vol. 8 (1957) pp. 598-601.

4. A. Marx, Untersuchungen ibber schlichte Abbildungen, Math. Ann. vol. 107 (1932-1933) pp. 40-67.

5. Polya und Szegö, Aufgaben und Lehrsätze, vol. 1, problem 287, p. 140.

6. M. S. Robertson, On the theory of univalent functions, Ann. of Math. vol. 37 (1936) pp. 374-408.

7. A. Schild, On a class of functions schlicht in the unit circle, Proc. Amer. Math. Soc. vol. 5 (1954) pp. 115-120.

8. E. Strohäcker, Beitrage zur Theorie der schlichten Funktionen, Math. Z. vol. 37 (1933) pp. 356-380.

TEMPLE UNIVERSITY 